\title{
Conjugated Oligonucleotides for Biochemical Applications
}

\author{
Massimo L Capobianco ${ }^{1 *}$, Maria Luisa Navacchia1, Elena Marchesi² and Daniela Perrone ${ }^{2}$ \\ 1 Istituto per la Sintesi Organica e la Fotoreattività del Consiglio Nazionale delle Ricerche (ISOF-CNR), Bologna, Italy \\ ${ }^{2}$ Dipartimento di Scienze Chimiche e Farmaceutiche, Università di Ferrara, Ferrrara, Italy
}

\begin{abstract}
In the last 30 years oligonucleotides i.e., relatively short polymers (usually 12-24 units long) based on DNA structure, have found a widespread use in biochemical studies and as biochemical probes and chemotherapeutic agents for the downregulation of genetic expression or for exon skipping. Here we present a short review of studies from our laboratories on the synthesis and applications of different kind of conjugates to address some of these techniques. Preparation of conjugates with small alkyl groups, intercalators, fluorescent oligothiophenes, and lipophilic bile-acids will be discussed.
\end{abstract}

Keywords: Conjugated oligonucleotides; Antisense; Anti-gene; Molecular probes

\section{Introduction}

Natural oligonucleotides, i.e., short fragments of single stranded DNA have been actively studied after the discovery of the significance of the double stranded DNA in living beings. Their chemical synthesis was attempted since the '60s after the pioneering works of Todd [1] and Khorana [2,3], based on a tedious and difficult repetition of chemical steps aimed at the addition of single nucleosides employing the methodology of active esters of $\mathrm{P}(\mathrm{V})[4,5]$. Despite the difficulties of this approach, short sequences of DNA with a known sequence were employed for structural characterization X-ray crystallography and NMR [6,7], studies of drugs-binding (distamycins [8], daunomycins [9] etc.), and as antisense gene down-regulators [10]. In the '80s, following the development of the intuition of Beaucage and Carruthers [11], a new strategy of synthesis based on the high reactivity of P(III) was developed [12] and found a widespread application toward the automated synthesis starting from the 3'-terminal nucleoside attached to a solid support normally controlled pore glass (CPG). Since then little has changed, but as chemists, we were challenged with the poor performance of exogenous DNA. In fact, outside of the strict cellular control, exogenous oligonucleotides encounter difficulties to cross the cell membranes, and once inside cells are rapidly degraded by nucleases before reaching the desired target. A first response to the fast degradation challenge was the development of oligonucleotides with modified backbones (phosphorothioates, phosphoridithioates, etc.), modified sugars (arabino, 2'-O-Alkyl-RNA) or both (morpholino, PNA etc.). In this rush, we discovered, despite numerous successful applications, the uniqueness of natural sequences, for the fine regulation of biochemical processes; in fact modified oligo's simply have too many chiral centers, the wrong solubility, and a too high or too low affinity toward their complementary sequences. A more sensible approach for dealing with such precious molecules is considering oligonucleotides as strings of "biochemical information" and try to perturb the natural structure with the "minimal efficient modification" that sometime, can be obtained with the chemical conjugation of small molecules.

\section{Antisense Stability}

The antisense methodology is a way to impair the genetic expression of an unwanted gene interfering with its translation forming a duplex with the sense RNA using an exogenous oligonucleotide (usually of DNA type). There are many good reviews on this methodology $[13,14]$ that can be carried out with several variations. In its basic form, the "antisense" oligonucleotide pairs according to Watson and Crick to the "sense" mRNA. This hetero-duplex can be degraded by RNase-H preventing the synthesis of the corresponding protein. Several factors complicate this nice picture; one of them is the degradation of the "antisense" especially by exonucleases, before it reaches its target. To overcome this problem, many researchers proposed the use of phosphorothioated oligonucleotides that have a good resistance against nuclease degradation. However, phosphorothioates have two serious drawbacks: they introduce one chiral phosphorous per substitution, so that an 18-mer PS oligo is a complex mixture of $2^{17}$ different diasteroisomers; secondly they tend to bind a-specifically to cellular proteins, causing unwanted side effects $[15,16]$. A more sensible approach seems that of using terminal modification to the antisense oligonucleotide. One of the first attempts in this direction was that of Zamecnik who in 1978 published a work on an oligonucleotide to be used as antisense agents against the Ros sarcoma virus with both ends protected as phenylcarbamoyl derivatives [10]. According to the procedure of Agarwal [17] the protected tridecamer prepared with the phosphotriester method [4] was reacted, in its protected form but with 5' and 3' free hydroxyl groups, with phenylisocyanate to give after deprotection the end capped phenylcarbamoyl-oligonucleotide. The efficiency of the protection can be tested against the snake venom phosphodiesterase (SVPDE) a 3'-exonuclease or calf-spleen phosphodiesterase (CSPDE) the corresponding 5 ' enzyme.

Following the Zamecknic's example, we prepared a set of oligonucleotides of identical sequence, but having the extremities conjugated with propandiol, hexandiol, or seco-uridine (Figure 1) and compared them with phosphorothioated analogs toward resistance against 3 '- and 5 '- exonucleases. The efficiency of the conjugation was tested by HPLC against SVPDE and CSPDE finding an increase resistance toward both degradations of all the end-modified oligonucleotides, with a more than ten times increased resistance of the seco-uridine conjugates respect to the natural unproteted oligonucleotide [18].

The 5'-end seco-uridine modification, and similarly the propandiol and hexandiol derivatives, were obtained preparing the corresponding

${ }^{*}$ Corresponding author: Massimo L, Istituto per la Sintesi Organica e la Fotoreattività del Consiglio Nazionale delle Ricerche (ISOF-CNR), Bologna, Italy, Tel: +390516398287; Fax: +390516398349; E-mail: massimo.capobianco@isof.cnr.it

Received June 26, 2017; Accepted July 07, 2017; Published July 15, 2017

Citation: Capobianco ML, Navacchia ML, Marchesi E, Perrone D (2017) Conjugated Oligonucleotides for Biochemical Applications. Med Chem (Los Angeles) 7: 197-207. doi: 10.4172/2161-0444.1000457

Copyright: (c) 2017 Capobianco ML, et al. This is an open-access article distributed under the terms of the Creative Commons Attribution License, which permits unrestricted use, distribution, and reproduction in any medium, provided the original author and source are credited. 


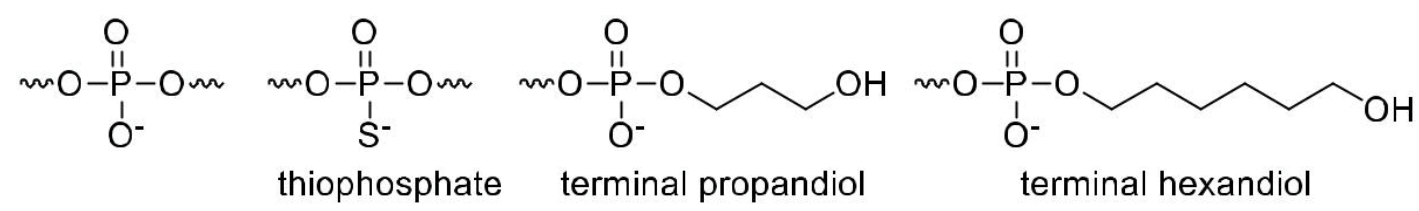<smiles>O=c1ccn(C(CO)OC(CO)COP(=O)([O-])O[Na])c(=O)[nH]1</smiles><smiles>COP(=O)([O-])OCC(CO)OC(CO)n1ccc(=O)[nH]c1=O</smiles>

\section{3'-seco-uridine modification}

Figure 1: Terminal modifications. The amidites and the modified solid support (CPG) necessary for the preparation of the end protected oligonucleotides were prepared in analogy with the chemistry reported in Scheme 1.

phosphoramidites, suitable for the automated synthesis, while for the 3 '-end modification we had to derivatize the used solid support reacting the aminoalkyl-CPG with the dimethoxytrityl protected uridine with succinic anhydride and a condenser. The logic behind the preparation of those compounds is sketched in Scheme 1. Briefly: uridine $\mathbf{1}$ was protected selectively at the 5 ' position with 4,4'-dimethoxytrityl chloride in pyridine to give $\mathbf{2}$ that was oxidized to the dialdehyde $\mathbf{3}$ with sodium periodate in water/acetone; 3 was reduced with sodium borohydride in ethanol to $\mathbf{4}$ that was converted to the monobenzoyl ester mixture $5+6$ with one equivalent of benzoyl chloride in pyridine. The mixture was separated on silica gel and compound $\mathbf{5}$ was used for the preparation of amidite 7 by use of 2-cyanoethyl- $N, N$-diisopropylchlorophosphorami dite in dichloromethane and 5 eq. of diisopropylethylamine (DIPEA). The mixture $\mathbf{5}+\mathbf{6}$ was used to prepare the succinic ester by reaction with the corresponding anhydride, and dicyclohexylcarbodiimide (DCC) as a condenser, in pyridine, then the crude filtrate was reacted with long chain amine-CPG $(n=6)$ or amine-CPG $(n=3)$ by gentle shaking in a sealed vial to the modified CPG 8 , finally capped with acetic anhydride.

\section{Exon Skipping}

A variant of the antisense approach is that of exon skipping. Antisense-mediated exon skipping [19] is a promising approach for the treatment of Duchenne Muscular Dystrophy DMD, a rare genetic disease due to a mutation of the dystrophin gene that cause the production of a truncated not functional protein. Targeting the antisense oligonucleotide to a selected region of the pre-mRNA, it is possible to modify the normal introne-hexone reading frame in a way to exclude the sequence that during the translation codes for a stop signal, in favor of a shorter but still partly functional variant of dystrophin. For this application, that do not require the intervention of RNase-H, 2'OMe-RNA oligonucleotides are normally used, in the form of phosphorothioates (PS). Among other factors, the efficient delivery of the oligonucleotide remains a significant challenge for its use as therapeutic drugs, and we demonstrated that a possible solution was to convey the oligonucleotides across the cell membrane through the use of cationic core-shell nanoparticles [20,21].

A new possibility raised some years ago when Regen and coll. conjugated a 5'-thiol modified T18 with two molecules of bile acids joined by a flexible scaffold [22,23]. They found that this assembly allowed the bile acids hydrophilic faces to wrap around the oligonucleotide presenting the opposite lipophilic surfaces toward an artificial liposomal membrane, simulating a cellular one helping the conjugated oligonucleotide to cross the bilayer membrane, with the ingenious mechanism (dubbed molecular umbrella) depitched in Figure 2.

Mimicking this approach, we are now testing a 2'OMe-RNA-PS oligonucleotide targeting exon 23 in mdx mouse, provided with a tri-valent bile acid scaffold (Scheme 2). To this aim, a post-synthetic conjugation approach was assessed, i.e., during the solid phase synthesis, the oligonucleotide was equipped at the 5 -end with the appropriate functionality to which the lipophilic moiety was coupled in solution after release and purification of the antisense. Firstly, we have prepared the hydrophobic moiety. Thus, the known $3 \alpha-\mathrm{N}_{3}$-ursodeoxycholic methyl ester ( $\left.3 \boldsymbol{\alpha N}_{3} \mathbf{U D C}\right)$ [24] and the tris-propargyloxyamino derivative 9 obtained from TRIS according to a literature procedure [25] were chosen as building blocks for its synthesis. For assembling the desired tri-valent bile acid scaffold 10 we used the copper-catalyzed azide-alkyne cycloaddition (CuAAC) under microwave irradiations. In these conditions we isolated $\mathbf{1 0}$ in $24 \%$ overall yield after flash chromatography. Compound 10, was subjected to $N$-succinylation with succinic anhydride, and then transformed into the reactive ester $\mathbf{1 1}$ by using $N$-hydroxysuccinimide (NHS) in presence of DCC, with an overall yield of $83 \%$. Finally, the conjugation of the 5 -amino-modified antisense $\left(\mathbf{5}^{\prime}-\mathrm{NH}_{2}-\mathbf{A O N}\right)$ with the lipophilic $N$-hydroxysuccinimide ester 11 was accomplished in solution, by carrying out the reaction in bicarbonate buffer at $\mathrm{pH} 8.2$ for 20 hours to achieve the desired lipophilic oligonucleotide $\mathbf{1 2}$ with $30 \%$ yield after reverse phase purification. The ability of the new synthesized lipophilic oligonucleotide $\mathbf{1 2}$ to cross the phospholipid membrane is currently underway in our laboratories.

\section{Anti-gene Studies with Oligonucleotide-Daunomycin Conjugates}

The anti-gene approach, i.e., the use of an oligonucleotide to interfere with the transcription of the DNA and ultimately with the expression of the encoded protein, arises from the discovery of the possibility of binding an oligonucleotide to some regions of a regular duplex. In 1957, 


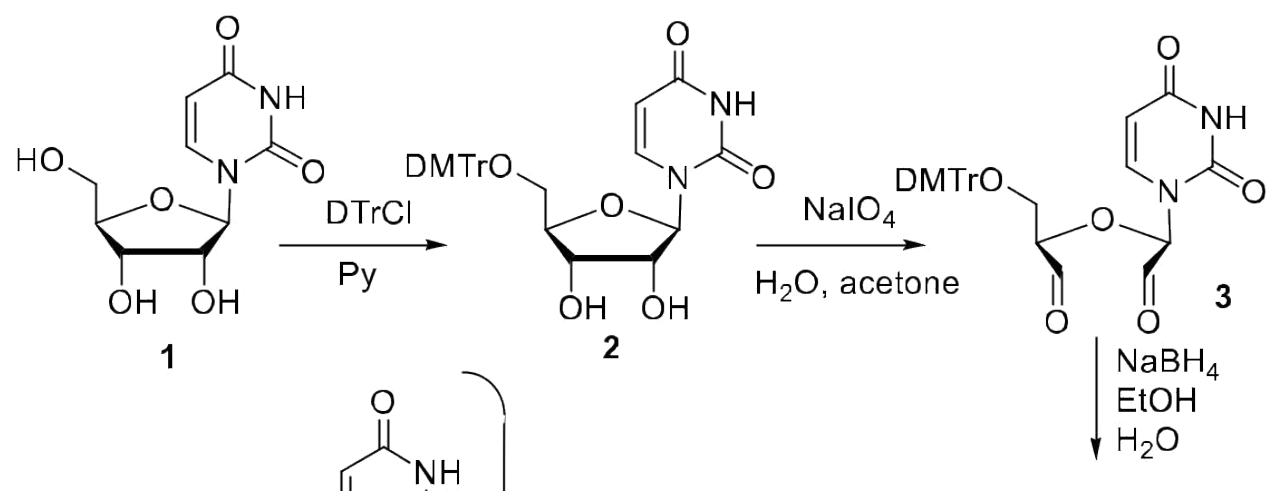

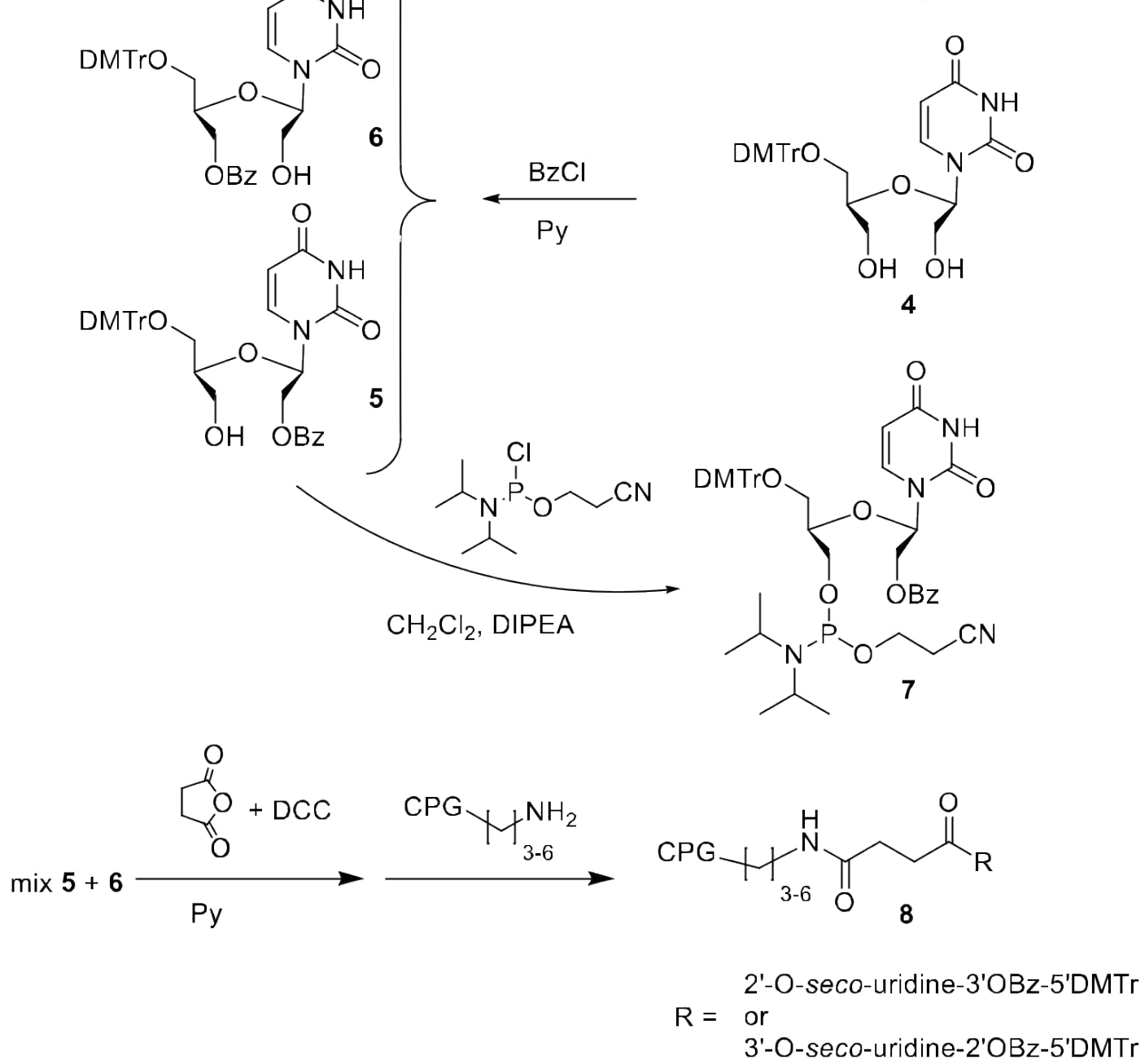

Scheme 1: Synthesis of the 5'-seco-uridine modification (compound 7), and of the loaded CPG (compounds 8) for the 3'-seco-uridine modification.

in fact, Felsenfeld found evidences of the existence of a three stranded structure of composition 2PolyU:polyA [26]; several years later Lyamichev published the "Structures of homopurine-homopyrimidine tract in superhelical DNA"1 [27] then called H-DNA. When it was clear that the resulting "triplex" complex was formed by an oligonucleotide located into the major groove of the DNA, binding the homopurinic strand, through Hoogsteen or reverse Hoogsteen hydrogen bond patterns, the gene itself became a target for several kind of biochemical applications. Pioneers in this field were Dervan [28] and Hélène [29]. At difference with what happen by using oligonucleotides as antisense agents, in the triplex approach the oligonucleotide is designed to bind directly to the gene of interest, inside the nucleus of eukaryotic cells. The theoretical tremendous advantage of such a technique over the antisense methodology is evident from the count of the target molecules, in principle a single molecule could shut down a gene in a single cell. Moreover it was demonstrated that the approach is feasible despite the presence of chromatine [30]. The success of this methodology depends among other factors (cellular uptake, biodistribution, stability etc.) on the strength of binding of the triplex forming oligonucleotide (TFO) to its target, and several groups used to conjugate the TFO with DNA 

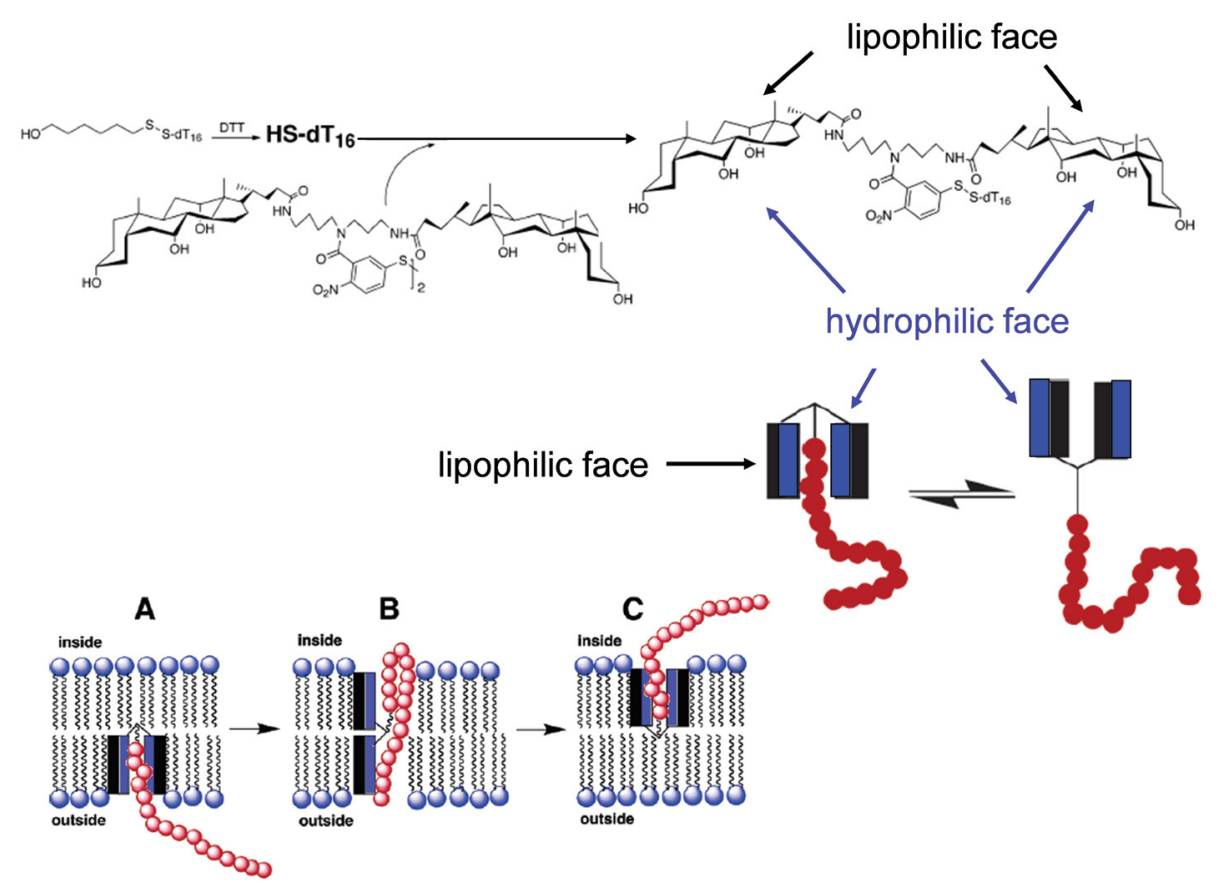

Figure 2: Scheme of synthesis and representation of the crossing of a bilayer phospholipid membrane aided by the "umbrella effect". Reprinted adapted with permission from: $[22,23]$<smiles>[X]COCC(N)(COC[X])COC[X]</smiles><smiles>[X]COCC(COC[X])(COC[X])NC(=O)CCC(=O)ON1C(=O)CCC1=O</smiles>

Scheme 2: Synthesis of a tri-ursodeoxycholic 2'OMeRNA-PS oligonucleotide conjugate 12. a. CuSO (4 mol\%)/ascorbate $(20 \mathrm{~mol} \%)$ in ${ }^{\mathrm{B}} \mathrm{BuOH} / \mathrm{H}_{2} \mathrm{O}(4: 1)$ microwave irradiations $\left(80^{\circ} \mathrm{C}, 45 \mathrm{~min}\right),\left(24 \%\right.$ yield); b. succinic anhydride $(2 \mathrm{eq}), \mathrm{Et}_{3} \mathrm{~N}(0.1 \mathrm{eq})$, in $\mathrm{CH}_{2} \mathrm{Cl}_{2} / \mathrm{THF}^{\mathrm{H}}(2: 1), 6 \mathrm{~h} 60^{\circ} \mathrm{C}$, (90\% yield); c. NHS (1.2 eq), DCC (1.2 eq), in $\mathrm{CH}_{2} \mathrm{Cl}_{2}, 12 \mathrm{~h} \mathrm{rt},\left(90 \%\right.$ yield); d. 5'- $\mathrm{NH}_{2}-\mathrm{AON}$, in $\mathrm{NaHCO}_{3} \mathrm{pH} 8.2,20 \mathrm{~h} \mathrm{rt},(30 \%$ yield). 
intercalators to enhance the stability of the triplex aggregate [31]. We decided to follow this strategy employing daunomycin. Daunomycin is a powerful antitumor molecule that interact with DNA, intercalating its anthraquinone planar moiety between two couples of bases (preferentially $\mathrm{GpC}$ ) laying the aminosugar into the minor groove [32]. Following this intercalation, its natural fluorescence is quenched, (this can be monitored to demonstrate the binding), and the complex DNAdaunomycin prevents the topoisomerase II resealing after the cleavage of one of the strands required by the unwinding, eventually leading to cellular death.

Some groups had already performed this conjugation binding the TFO with the aminogroup of the glycone of the daunomycin [33], but with little gain in terms of triplex stabilization and biological effect. We thought that better results could be achieved binding the daunomycin to the TFO at the opposite end, to preserve the natural way of intercalation of daunomycin with DNA, since the TFO binds the homopurinic strand displaying itself into the major groove of the duplex. Following this idea, we made a partnership with researchers of Menarini Laboratories, who helped us to modify the daunomycin. Ultimately we settled on a procedure based on the attachment of a iodohexametylene chain on the $\mathrm{O}-4$ of the daunomycin and a post synthetic conjugation of the oligonucleotide via a chemoselective binding of a terminal thiophosphate group of the TFO [33] as depitched in Scheme $3[34,35]$ the ${ }^{5} \mathrm{PS}-\mathrm{TFO} 14$ was prepared using a $0.1 \mathrm{M}$ acetonitrile solution of bis-2-cyanoethyl( $N, N$-diisopropyl)-phosphoramidite [36] with a longer coupling time on the automated synthesizer to ensure the complete coupling, followed by thio-oxidation, we get the PSunprotected oligo after usual ammonia deblocking. The ${ }^{31} \mathrm{P}-\mathrm{NMR}$ allowed us to judge if the oligonucleotide had the right $\mathrm{PS} / \mathrm{PO}$ ratio. The conjugation with the protected daunomycin 13 was performed in water/DMF. After water/dichloromethane extraction only the conjugate 15 remains in water. The protective groups on the amino sugar were removed by reaction with an aqueous diluted soda solution; finally, the unprotected oligo $\mathbf{1 6}$ was purified by preparative reversed phase HPLC. The progress of the reaction could easily be monitored by HPLC, moreover the ${ }^{31} \mathrm{P}-\mathrm{NMR}$ signal of the phosphorothioate changed from $46 \mathrm{ppm}$ of the monoester derivative $\mathbf{1 4}$ to $23 \mathrm{ppm}$ of compound 16 (and 15). Later on we were able to prepare daunomicyn conjugates also in the $3^{\prime}$ position (and in both $5^{\prime}$ and $3^{\prime}$ position) preparing a modified CPG according to a published procedure [37]. We utilized this modified CPG to prepare 3'-PS oligonucleotides [38] and from this a 3 -conjugate. Daunomiycin-TFO conjugates were applied with some interesting results against the poly purine tract of HIV-1 and against the promoter $\mathrm{P} 2$ of c-myc oncogene [35]. In the end, the efforts spent in this research leaded, as hoped, to a demonstration of an active role of the conjugated daunomycin after intercalation, mediated by topoisomerase. Indeed, targeting the same site MDR1 in resistant cells lines (MCF7-R and NIH-3T3) with a 5 ' or a 3 ' daunomycin-TFO conjugate, we observed the higher biological response with the less stable of the pair [39].

\section{Fluorescent Probes}

We had demonstrated for the first time the compatibility of oligothiophenes as fluorescent markers for oligonucleotides Scheme 4 [40].

The ready available terthiophene alcohol 17 can be reacted with the 2-cyanoethyl- $\mathrm{N}, \mathrm{N}$-diisopropylchlorophosphoramidite to give the corresponding amidite $\mathbf{1 8}$ that was found suitable for the conventional solid phase synthesis of oligonucleotides. We prepared the showed tetrathymidine conjugate 19 characterized by NMR, UV and fluorescence. Unlikely the conventional "old-fashionable" fluorescein labeled conjugates, these derivatives are stable under UV light without showing any bleaching.

The developments of those fluorescent labels made possible the design of biological probes in the form of molecular beacons (MBs). MBs are oligonucleotides bearing a fluorophore at one end and a quencher on the other. They are designed with short self-complementary regions flanking a central recognition sequence. In absence of a target the flanking regions pair, putting the fluorophore and the quencher in close proximity, in a conformation that graphically resemble to a hairpin; in that form the fluoresce is reduced by fluorescence resonance energy transfer (FRET). Conversely in presence of a target complementary to the central sequence, the MB forms a duplex with the target, the quencher is distanced from the fluorophore (more than the Förster distance) and the fluorescence is restored [41] (Figure 3).

We tried to prepare MBs employing oligothiophenes, thus we synthesized a 5'-dabcyl conjugate oligonucleotide with a free 3 '-amino group. From this, we prepared several different oligothiophene derivatives using the corresponding succinimide-oligothiophene (Figure 4 left panel). Surprisingly we found that to detect the complementary target the presence of a quencher was not strictly necessary [42,43] (Figure 4 right panel), probably because this kind of

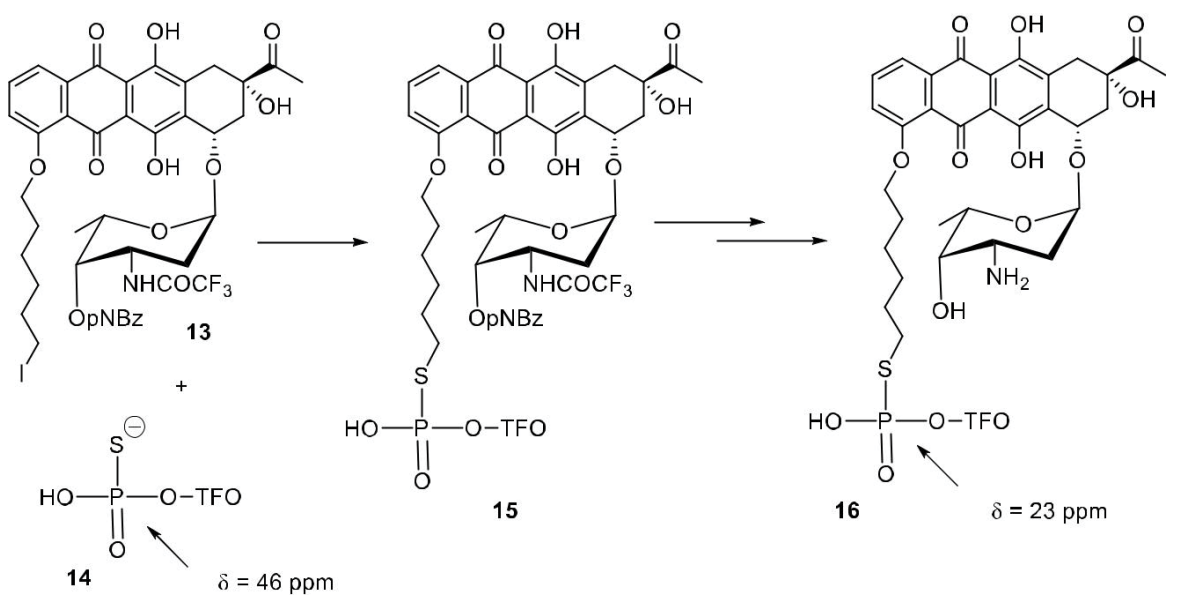

Scheme 3: Synthesis of daunomicyn-TFO conjugate (16) as down-regulator of genetic expression through triplex formation 


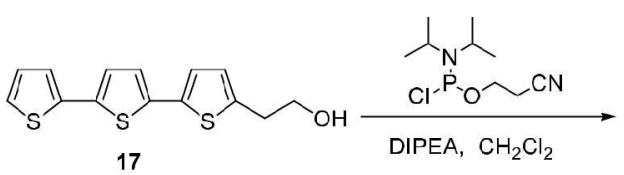

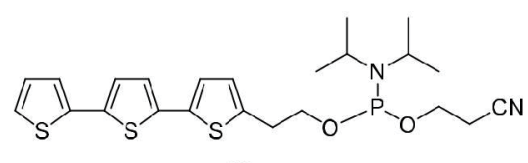

18

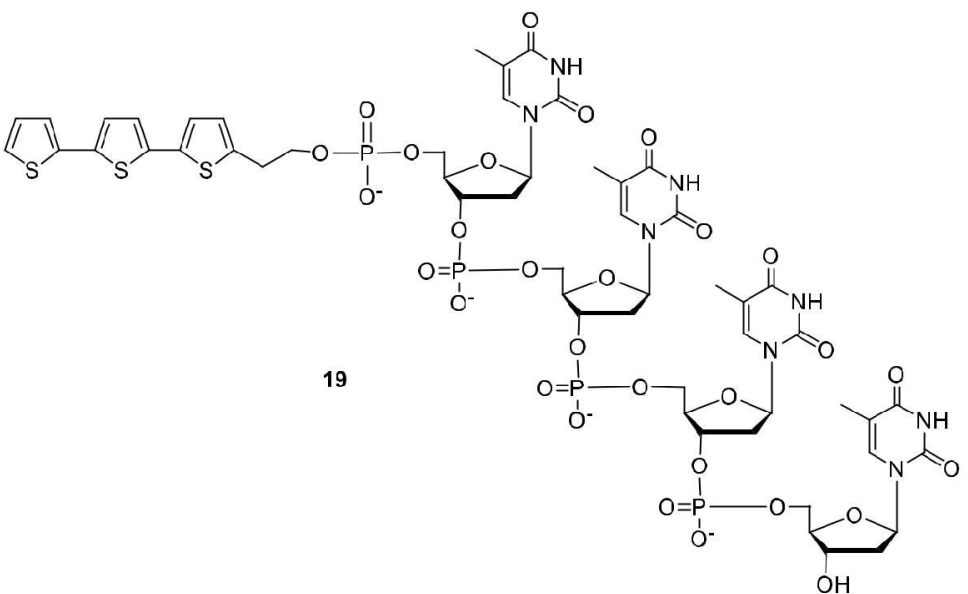

Scheme 4: The synthesis of the first fluorescent oligothiophene-oligonucleotide conjugate (19).

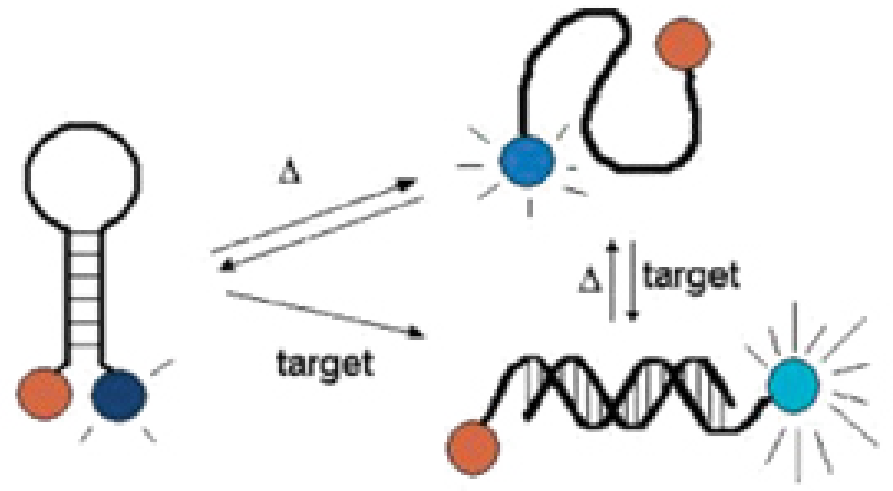

Figure 3: Sketch of the functioning of a molecular beacon (MB). The hairpin structure puts the fluorophore and the quencher in close proximity so that the fluorescence is strongly reduced. Fluorescence is restored after thermal denaturation or hybridization of the MB with a complementary target.
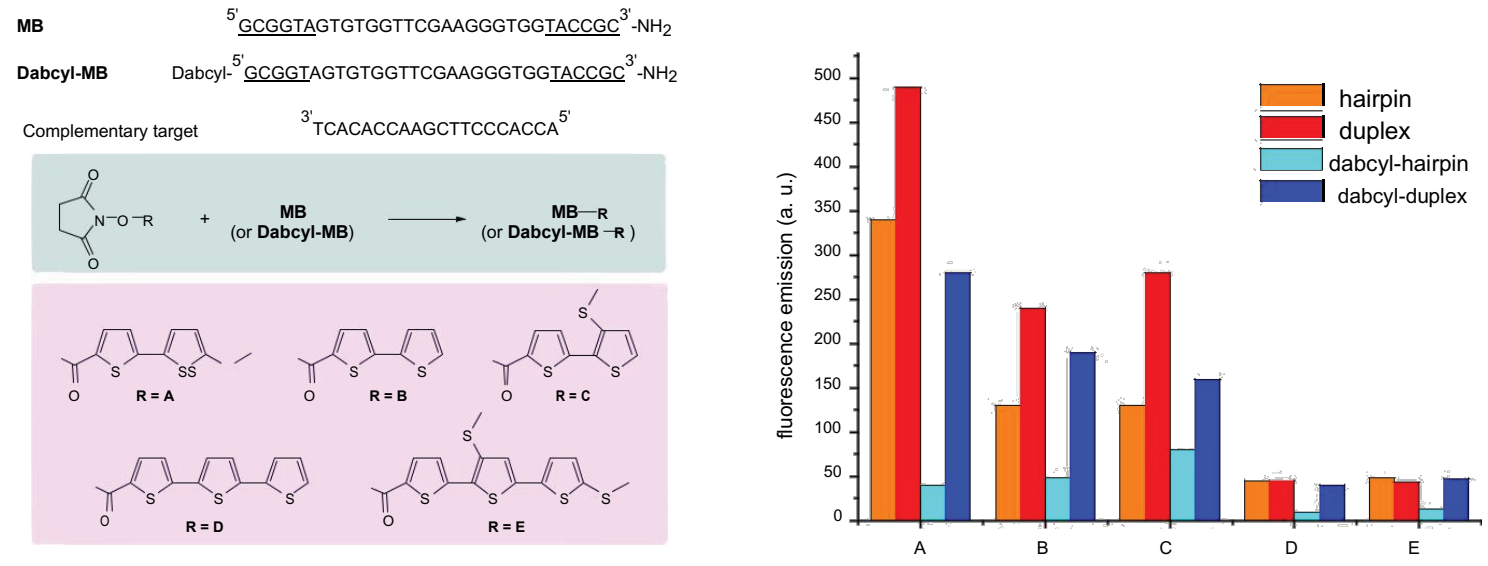

Figure 4: Left panel: sequences, methodology of synthesis and structure of the fluorophores. Right panel: emitted fluorescence of the corresponding hairpin or duplex with or without the dabcyl quencher. 
fluorescent tags were able to exchange energy by resonance with the guanines at the opposite ends.

Oligothiophene fluorophores can be electronically coupled to a uridine base to make it sensible to the correct base pairing, then used to detect single nucleotide mutations [43,44] (Figure 5).

In this case, the uridine derivatives (20-23) can be seen as conjugate nucleosides having an oligothiophene joined to the uridine base with a conductive linker. The formation of a hydrogen bonding following the correct pairing (U:dA) modifies the molecular orbitals of the uridine and of the conjugate label, resulting in a different fluorescence. We found that the phosphoramidite of the 5'-dimethoytrityl modified uridines (20-23) were suitable for the automated synthesis of oligonucleotides and were not affected by the following deblocking procedures. The resulting oligonucleotides containing the modified uridine at the middle of the sequence, showed appreciable fluorescence variations when faced with an oligonucleotide containing the correct or a mismatched base (Figure 5 right panel).

Compounds 20-23 were prepared via Sonogashira's coupling (20) or reverse coupling $(\mathbf{2 1})$ or from Stille's coupling reaction $(\mathbf{2 2}, \mathbf{2 3})$ starting from 5'-dimethoxytrityl-5-iodouridine $\mathbf{2 4}$ or 5'-dimethoxytrityl-5etinyl-uridine (26) according Scheme 5. The amidites were prepared by reaction of the corresponding precursors with 2 -cyanoethyl- $N, N$ -

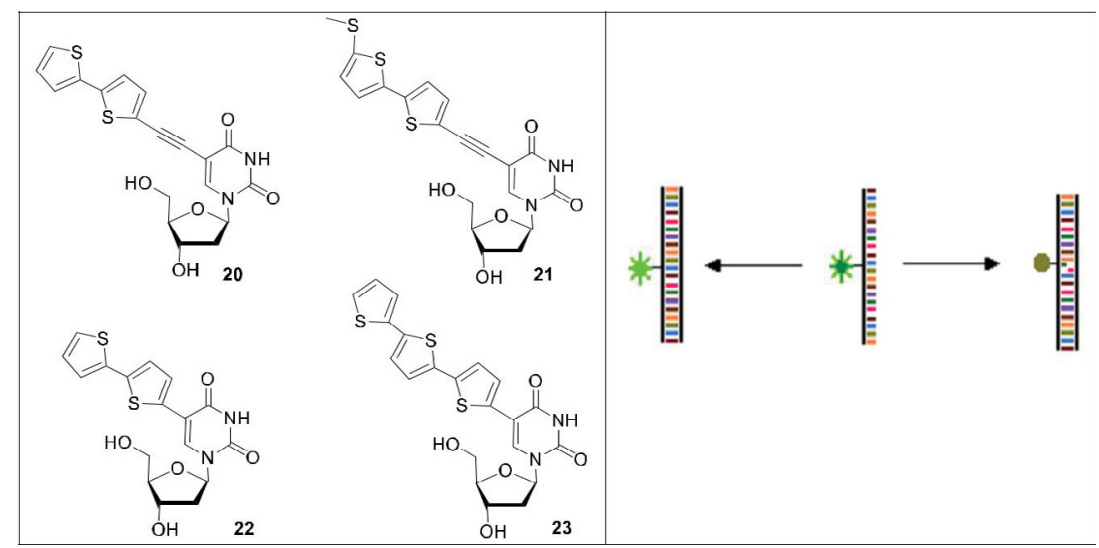

Figure 5: Left panel: modified uridines used to detect single nucleotide polymorphisms. Right panel: hybridization experiments; the probe sequence containing the modified uridine in the middle, showed a different fluorescence pattern when hybridized with a complementary strand containing a deoxy-adenosine, or any other base in the facing position.

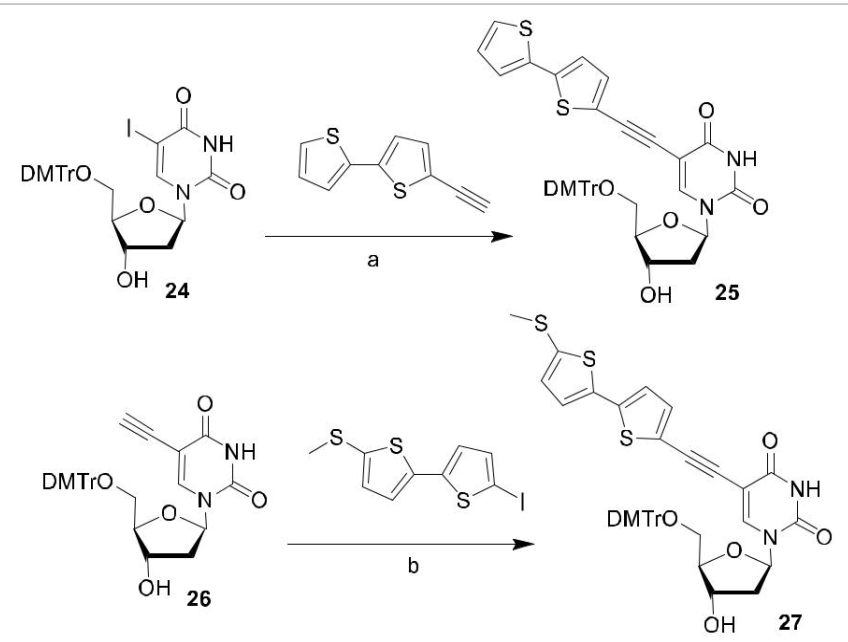

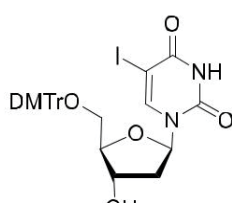

$\mathrm{OH} 24$
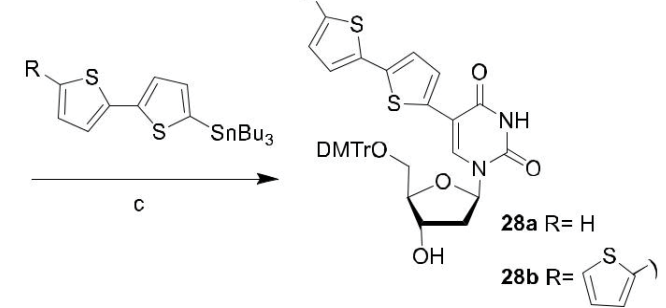

Scheme 5: Synthesis of compounds 25-28 containing the modified uridine 20-23 utilized for the SNP detection. a. Sonogashira's coupling Pd(PPh3)4, Cul, TEA in DMF, $3 \mathrm{~h} \mathrm{rt}$, (60\% yield); b. Reverse Sonogashira's coupling Pd(PPh3)4, Cul, TEA in DMF, $3 \mathrm{~h} \mathrm{rt},\left(66 \%\right.$ yield); c. Stille's coupling: Pd(PPh3)2Cl2 in dioxane, $4 \mathrm{~h} 95^{\circ} \mathrm{C}$, (yields: $17 \mathrm{a} 97 \%, 17 \mathrm{~b} 40 \%)$. 
diisopropylchlorophosphoramidite in dichloromethane and DIPEA (in case of compound 25) or with the bis- $N, N$-diisopropyl(2-Ocyanoethyl)phosphite in dichloromethane/acetonitrile with one eq. of tetrazole (compounds 27 and 28a and b), in these cases isolation of the derivatives was not necessary.

\section{Adenosine Derivatives}

The possibility to bind a functional molecule to a nucleoside, as demonstrated above, offer the chance of precisely locating that molecule along an oligonucleotide, therefore enhancing the possibility of tuning a possible biochemical effect. Looking for a convenient way to use oligonucleotide conjugated with psoralene having the paper of Sproat [45] as a starting model, we settled on a versatile synthesis based on the modification of the C-8 position of deoxyadenosine. Psoralene is a planar molecule able to intercalate preferentially among TA base pairs to give, after UV irradiation, a reversible [2+2] cycloadduct with the C5-C6 atoms of the thymidine rings on the two facing strands. This molecule had been conjugated to antisense DNA since the beginning of this methodology [46,47]. Our original methodology was based on the efficient $\mathrm{C} 8$ alkylation of the adenine ring by a sulfur atom at the end of a bifunctional dithiol alkyl linker (Scheme 6). The 8-Brdeoxyadenosine $\mathbf{2 9}$ was reacted with an alkyl dithiol in hot water and few equivalents of TEA to give, after extraction with ethyl acetate, the derivative 30. This was readily converted to the psoralen nucleoside 31, then, through reactions c-e, it was $5^{\prime}$ protected with $\mathrm{DMTrCl}$ in pyridine to give compound $\mathbf{3 2}$, converted to $\mathbf{3 3}$ with diphenylacetyl chloride, and eventually to the corresponding amidite $\mathbf{3 4}$ by reaction with 2-cyanoethyl- $\mathrm{N}, \mathrm{N}$-diisopropylchlorophosphoramidite in dichloromethane and TEA. The psoralen nucleoside was finally inserted in the middle of a sequence by conventional solid phase synthesis.

The conjugated oligonucleotide containing the psoraleneadenosine nucleoside $\mathbf{3 1}$ was demonstrated to be able to bind to its complementary sequences and to do cross-linking upon UV irradiation by HPLC experiments (Figure 6) [48]

Utilizing the same chemistry, we were able to prepare a conjugate with a different intercalator, i.e., acridine (Scheme 7).

In this case the 2,9-dichloro-6-mehoxyacridine was reacted with 8-dithiopentenyldeoxyadenosine (compound $\mathbf{3 0}$ from the previous scheme), in DMF and TEA at $80^{\circ} \mathrm{C}$, for $17 \mathrm{~h}$ to give 35 (70\% yield). With the same reaction pattern c-e already seen this compound was tritylated to $\mathbf{3 6}$, protected with diphenylacetyl to 37 and eventually converted to the phosphoramidite $\mathbf{3 8}$ in analogy with the synthesis of the psoralene phosphoramidite $\mathbf{3 4}$ with similar yields. The oligonucleotide conjugated with this fluorescent molecule was found to have a reversible binding toward gold nanoparticles, possibly opening the way for a fast purification methodology for every oligonucleotides bearing at least two different sulfur atoms [49].

One interesting way to prepare a large variety of oligonucleotide conjugates is based on the orthogonal chemoselectivity of the clickchemistry. This kind of reaction can be performed with two partners, one having an azido group and the other a terminal alkyne. The reaction can be carried on in an aqueous solution and is largely independent from the presence of many chemical groups, so it is ideal to further elaborate complicate (bio)molecules. Again, our modification at the C- 8 of the 2'deoxyadenosine can be utilized to prepare oligonucleotides bearing an alkyne moiety as in Scheme 8 .

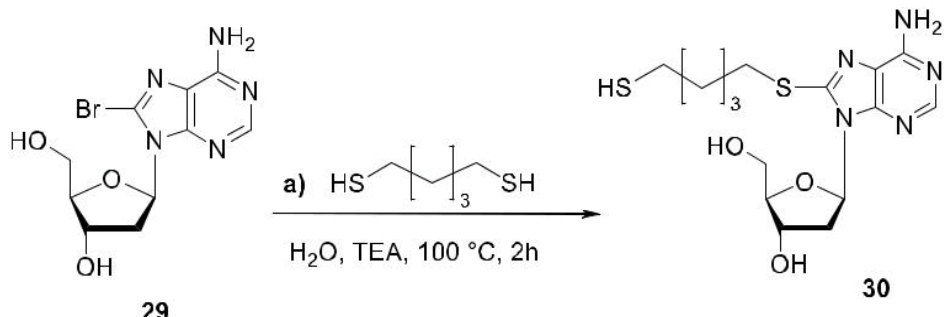

29
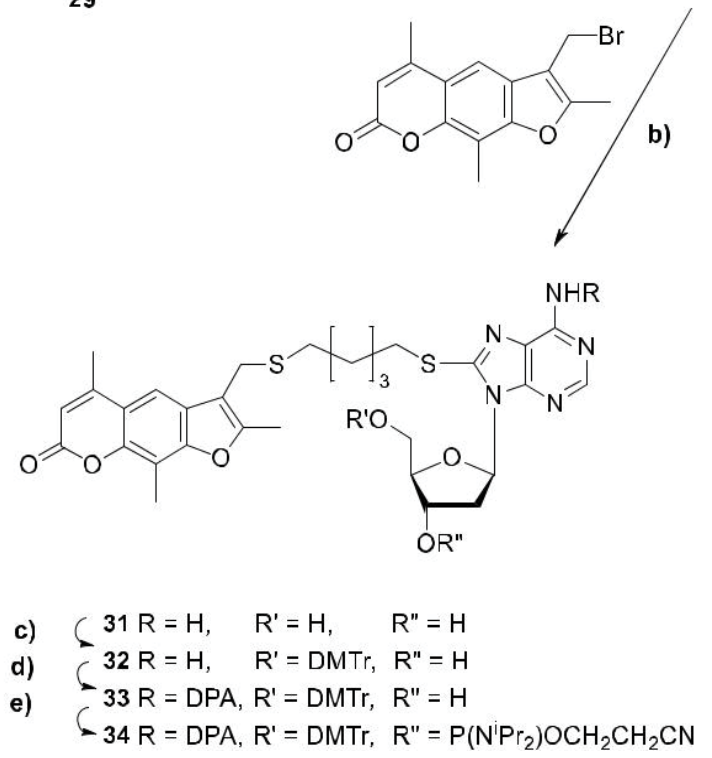

Scheme 6: Synthesis of psoralene-adenosine conjugates 31-33 and the corresponding amidite 34 . 


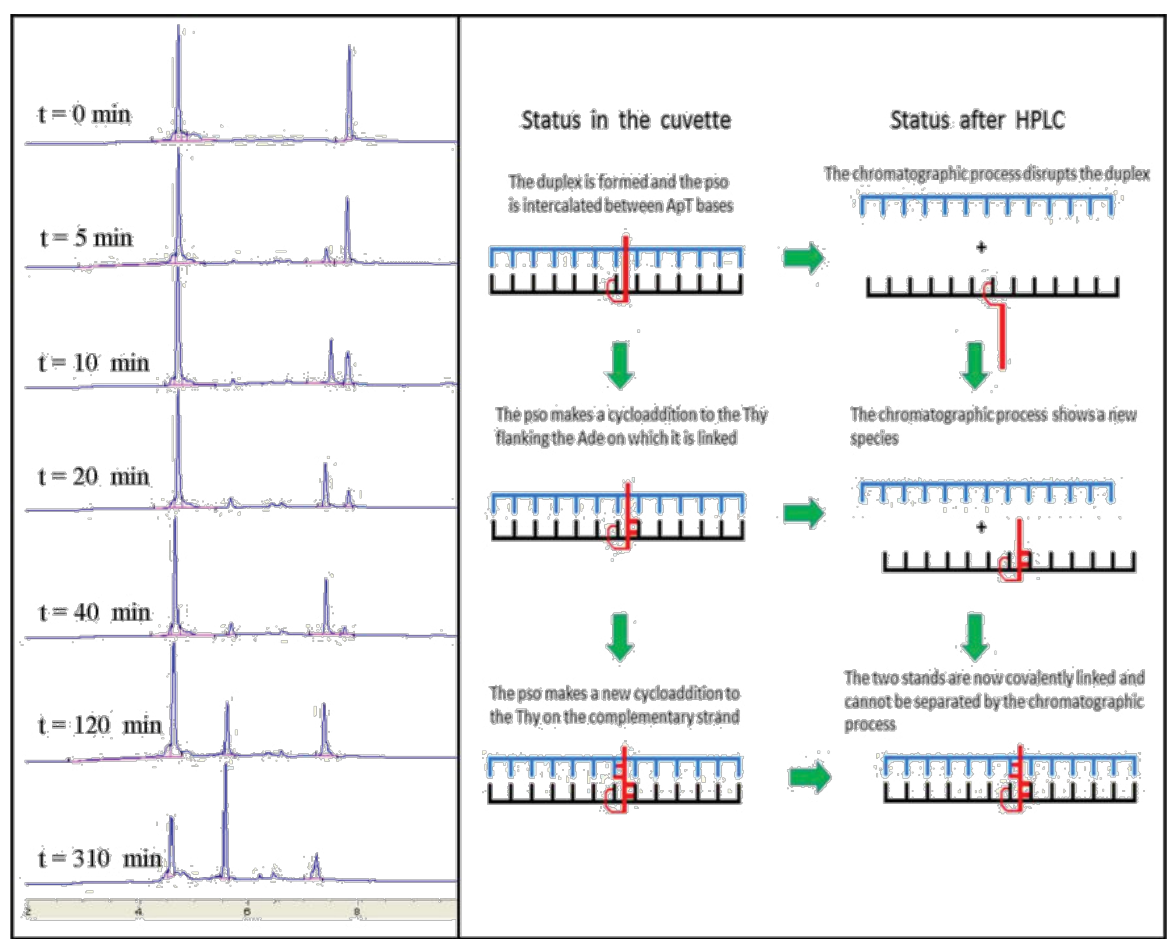

Figure 6: Left panel: HPLC profile of the UV irradiated mixture of the oligonucleotide-psoralen conjugate with its complementary DNA strand at different times. Right panel: interpretation of the chromatographic peaks.
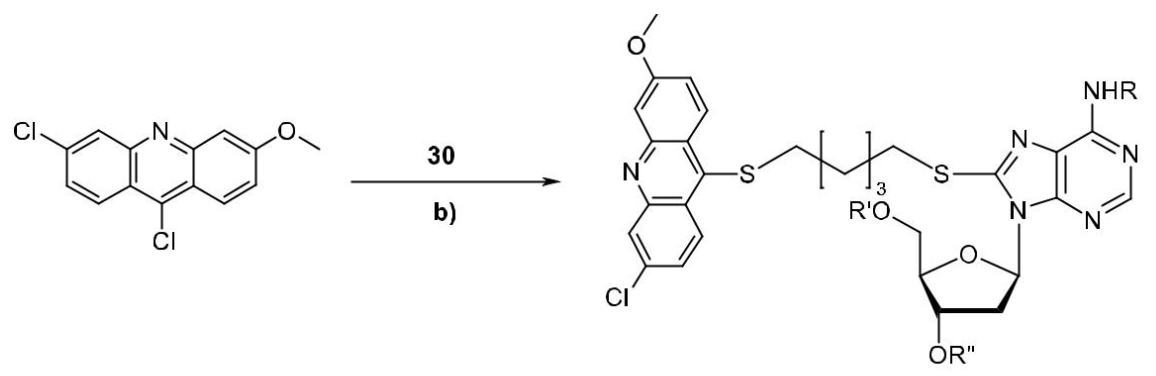

c) $\left(35 \mathrm{R}=\mathrm{H}, \quad \mathrm{R}^{\prime}=\mathrm{H}, \quad \mathrm{R}^{\prime \prime}=\mathrm{H}\right.$

d) $36 \mathrm{R}=\mathrm{H}, \quad \mathrm{R}^{\prime}=\mathrm{DMTr}, \mathrm{R}^{\prime \prime}=\mathrm{H}$

e) $37 \mathrm{R}=\mathrm{DPA}, \mathrm{R}^{\prime}=\mathrm{DMTr}, \mathrm{R}^{\prime \prime}=\mathrm{H}$

e) $\quad \begin{aligned} & 37 \mathrm{R}=\mathrm{DPA}, \mathrm{R}^{\prime}=\mathrm{DMTr}, \mathrm{R}^{\prime \prime}=\mathrm{H} \\ & 38 \mathrm{R}=\mathrm{DPA}, \mathrm{R}^{\prime}=\mathrm{DMTr}, \mathrm{R}^{\prime \prime}=\mathrm{P}\left(\mathrm{N}^{\prime} \mathrm{Pr}_{2}\right) \mathrm{OCH}_{2} \mathrm{CH}_{2} \mathrm{CN}\end{aligned}$

Scheme 7: Synthesis of the amidite of the acridine-deoxyadenosine conjugate.

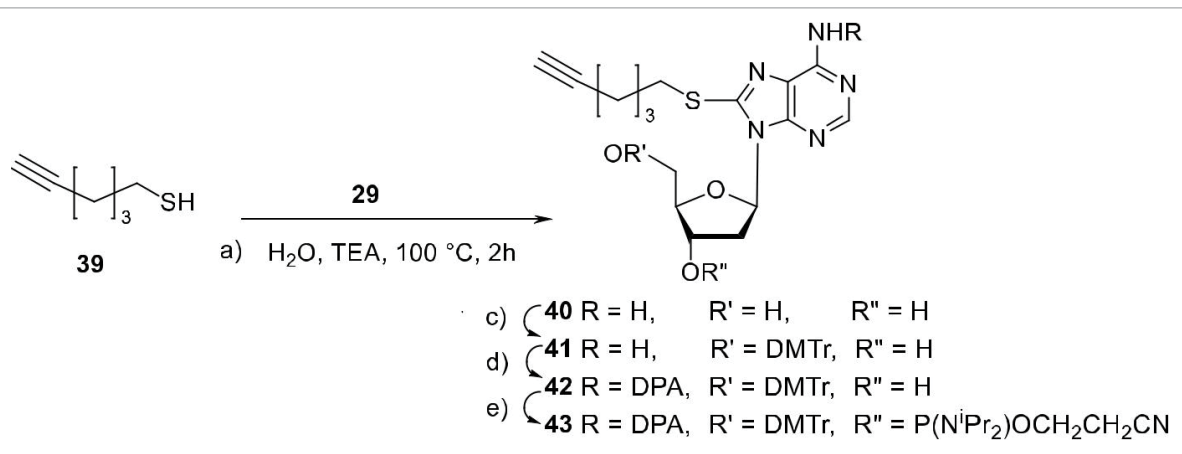

Scheme 8: Synthesis of the amidite 43 , a useful reagent to introduce a terminal alkyne into an oligonucleotide. 


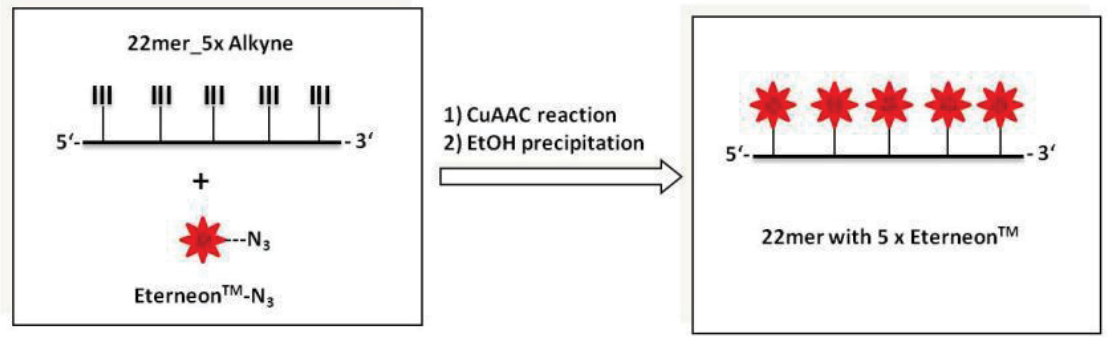

Figure 7: Preparation of a multiple fluorescent oligonucleotide using the click chemistry with commercially available reagents.

The synthesis of the compound proceeded as following: the 8-Brdeoxyadenosine 29 was reacted with the hex-5-yne-1-thiol 39 in water and TEA at $100{ }^{\circ} \mathrm{C}, 2 \mathrm{~h},(30 \%)$. Steps c-e were performed as in previous schemes. At the best of our knowledge the amidite of this derivative is the only alternative to the C6-pyrimidine analogues commercially available [50]. Through the click-chemistry these oligonucleotides can be further processed to the desired compound, connecting the desired tag to an azido-tether [48] even with multiple groups (Figure 7) [43].

\section{Conclusions}

The conjugation of small molecules to nucleic acids allows affording several techniques with the best aptly derivatives. The only limits are the imagination of scientists and the feasibility of the involved chemical steps. We hope that the reported applications can be of inspiration to scholars facing biochemical challenges.

\section{Conflicts of Interest}

The authors declare no conflict of interest.

\section{References}

1. Michelson AM, Todd AR (1955) Nucleotides part XXXII. Synthesis of a dithymidine dinucleotide containing a 3': 5'-internucleotidic linkage. Journal of the Chemical Society 2632-2638.

2. Gilham PT, Khorana HG (1958) Studies on Polynucleotides. I. A New and General Method for the Chemical Synthesis of the C5"-C3" Internucleotidic Linkage. Syntheses of Deoxyribo-dinucleotides. Journal of the American Chemical Society 80: 6212-6222.

3. Gilham PT, Khorana HG (1959) Studies on Polynucleotides. V.1 Stepwise Synthesis of Oligonucleotides. Syntheses of Thymidylyl-(5' $\left.\rightarrow 3^{\prime}\right)$-thymidylyl-(5' $\left.\rightarrow 3^{\prime}\right)$-thymidine and Deoxycytidylyl-(5' $\left.\rightarrow 3^{\prime}\right)$-deoxyadenylyl-(5' $\left.\rightarrow 3^{\prime}\right)$-thymidine 2. Journal of the American Chemical Society 81: 4647-4650.

4. Katagiri N, Itakura K, Narang SA (1975) Use of arylsulfonyltriazoles for the synthesis of oligonucleotides by the triester approach. Journal of the American Chemical Society 97: 7332-7337.

5. Denny WA, Leupin W, Kearns DK (1982) Simplified liquid phase preparation of four decadeoxyribonucleotides and their preliminary spectroscopic characterization. Helvetica Chimica Acta 65: 2372-2393.

6. Drew HR, Wing RM, Takano T, Broka C, Tanaka S, et al. (1981) Structure of a B-DNA Dodecamer - Conformation and Dynamics. 1. Proceedings of the National Academy of Sciences of the United States of America 78: 2179-2183.

7. Coll M, Frederick CA, Wang AHJ, Rich A (1987) A Bifurcated HydrogenBonded Conformation in the D(A.T) Base-Pairs of the DNA Dodecamer D(CGCAAATTTGCG) and Its Complex with Distamycin. Proceedings of the National Academy of Sciences of the United States of America 84: 8385-8389.

8. Kopka ML, Yoon C, Goodsell D, Pjura P, Dickerson RE (1985) The MolecularOrigin of DNA Drug Specificity in Netropsin and Distamycin. Proceedings of the National Academy of Sciences of the United States of America 82: 1376-1380.

9. Quigley GJ, Wang AH, Ughetto G, Van Der Marel G, Van Boom JH, et al. (1980) Molecular structure of an anticancer drug-DNA complex: daunomycin

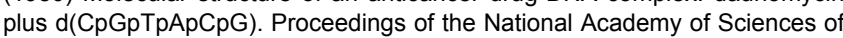
the United States of America 77: 7204-7208.
10. Zamecnick PC, Stephenson ML (1978) Inhibition of Rous sarcoma virus replication and cell transformation by a specific oligodeoxynucleotide. Proceedings of the National Academy of Sciences of the United States of America 75: 280-284.

11. Beaucage SL, Caruthers MH (1981) Deoxynucleoside phosphoramidites--A new class of key intermediates for deoxypolynucleotide synthesis. Tetrahedron Letters 22: 1859-1862.

12. Beaucage SL, lyer RP (1992) Advances in the Synthesis of Oligonucleotides by the Phosphoramidite Approach. Tetrahedron 48: 2223-2311.

13. Uhlmann E, Peyman A (1990) Antisense oligonucleotides: a new therapeutic principle. Chemical Reviews 90: 543-584.

14. Aboul-Fadl T (2005) Antisense oligonucleotides: the state of the art. Current Medicinal Chemistry 12: 2193-2214.

15. Zon G (1995) Antisense phosphorothioate oligodeoxynucleotides: Introductory concepts and possible molecular mechanisms of toxicity. Toxicology Letters 82: 419-424.

16. Krieg AM, Stein CA (1995) Phosphorothioate oligodeoxynucleotides: Antisense or anti-protein? Anisense Research Development 5: 241

17. Agarwal KL, Khorana HG (1972) Polynucleotides. ClI. Use of aromatic isocyanates for selective blocking of the therminal 3'-hydroxyl group in protected deoxyribooligonucleotides. Journal of the American Chemical Society 94: 3578-3585

18. Pandolfi D, Rauzi F, Capobianco ML (1999) Evaluation of different types of end-capping modifications on the stability of oligonucleotides toward 3 '- and 5 '-exonucleases. Nucleosides \& Nucleotides 18: 2051-2069.

19. Aartsma-Rus A, Van Ommen GJB (2007) Antisense-mediated exon skipping: A versatile tool with therapeutic and research applications. RNA 13: 1609-1624.

20. Rimessi $P$, Sabatelli $P$, Fabris M, Braghetta $P$, Bassi E, et al. (2009) Cationic PMMA Nanoparticles Bind and Deliver Antisense Oligoribonucleotides Allowing Restoration of Dystrophin Expression in the mdx Mouse. Molecular Therapy 17: $820-827$

21. Ferlini A, Sabatelli P, Fabris M, Bassi E, Falzarano S, et al. (2010) Dystrophin restoration in skeletal, heart and skin arrector pili smooth muscle of $\mathrm{mdx}$ mice by ZM2 NP-AON complexes. Gene Therapy 17: 432-438

22. Janout V, Regen SL (2005) A needle-and-thread approach to bilayer transport: permeation of a molecular umbrella-oligonucleotide conjugate across a phospholipid membrane. Journal of the American Chemical Society 127: 22-23.

23. Janout V, Jing B, Regen SL (2005) Molecular umbrella-assisted transport of an oligonucleotide across cholesterol-rich phospholipid bilayers. Journal of the American Chemical Society 127: 15862-15870.

24. Perrone D, Bortolini O, Fogagnolo M, Marchesi E, Mari L, et al. (2013) Synthesis and in vitro cytotoxicity of deoxyadenosine-bile acid conjugates linked with 1,2,3-triazole. New Journal of Chemistry 37: 3559-3567.

25. Chabre YM, Contino-Pepin C, Placide V, Shiao TC, Roy R, (2008) Expeditive synthesis of glycodendrimer scaffolds based on versatile TRIS and mannoside derivatives. Journal Organic Chemistry 73: 5602-5605.

26. Fenselfed G, Davies DR, Rich A (1957) Formation of a three-stranded polynucleotide molecule. Journal of the American Chemical Society 79: 20232024.

27. Lyamichev VI, Mirkin SM, Frank-Kamenetskii MD (1986) Structures of 
homopurine-homopyrimidine tract in superhelical DNA. Journal Biomolecular Structure Dynamic 5: 275-282.

28. Moser HE, Dervan PB (1987) Sequence-specific cleavage of double helical DNA by triple helix formation. Science 238: $645-650$.

29. Birg F, Praseuth D, Zerial A, Thuong NT, Asseline U, et al. (1990) Inhibition of simian virus 40 DNA replication in CV-1 cells by an oligodeoxynucleotide covalently linked to an intercalating agent., Nucleic Acids Research 18: 2901 2908.

30. Giovannangeli C, Diviacco S, Labrousse V, Gryaznov S, Charneau P, et al. (1997) Accessibility of nuclear DNA to triplex-forming oligonucleotides - the integrated HIV-1 provirus as a target., Proceedings of the National Academy of Sciences of the United States of America 94: 79-84.

31. Asseline U, Thuong NT, Helene C (1997) Synthesis and properties of oligonucleotides covalently linked to intercalating agents. New Journal of Chemistry 21: 5-17.

32. Wang AH, Ughetto G, Quigley GJ, Rich A (1987) Interactions between an anthracycline antibiotic and DNA: molecular structure of daunomycin complexed to $\mathrm{d}(\mathrm{CpGpTpApCpG)}$ at 1.2-A resolution. Biochemistry 26: 1152-1163.

33. Asseline U, Bonfils E, Kurfurst R, Chassignol M, Roig V, et al. (1992) SolidPhase Preparation of 5', 3'-Heterobifunctional Oligodeoxyribonucleotides Using Modified Solid Supports. Tetrahedron 48: 1233-1254.

34. Garbesi A, Bonazzi S, Zanella S, Capobianco ML, Giannini G, et al. (1997) Synthesis and binding properties of conjugates between oligodeoxynucleotides and daunorubicin derivatives. Nucleic Acids Research 25: 2121-2128.

35. Capobianco ML, Catapano CV (2008) Daunomycin-TFO conjugates for downregulation of gene expression: Anthracycline Chemistry and Biology II: Mode of Action, Clinical Aspects and New Drugs, Springer-Verlag Berlin Heidelberg

36. Uhlmann E, Engels J (1986) Chemical 5'-phosphorylation of oligonucleotides valuable in automated DNA synthesis. Tetrahedron Letters 27: 1023-1026.

37. Guzaev A, Lonnberg H, (1997) A novel solid support for synthesis of 3'-phosphorylated chimeric oligonucleotides containing internucleosidic methy phosphotriester and methylphosphonate linkages. Tetrahedron Letters 38 3989-3992.

38. Dembech C (2001) Sintesi e caratterizzazione di coniugati di oligonucleotid con derivati della daunorubicina formanti triple eliche, quali inibitori genici d c-myc: Degree Thesis, Facoltà di Farmacia, corso di laurea in CTF. University of Bologna.
39. Stierle V, Duca M, Halby L, Senamaud-Beaufort C, Capobianco ML, et al. (2008) Targeting MDR1 gene: Synthesis and cellular study of modified daunomycintriplex-forming oligonucleotide conjugates able to inhibit gene expression in resistant cell lines. Molecular Pharmacology 73: 1568-1577.

40. Capobianco ML, Naldi M, Zambianchi M, Barbarella G, (2005) Oligothiophene phosphoramidites for oligonucleotide labelling. Tetrahedron Letters 46: 8181 8184.

41. Tyagi S, Kramer FR, (1996) Molecular beacons: Probes that fluoresce upon hybridization. Nature Biotechnology 14: 303-308.

42. Cazzato A, Capobianco ML, Zambianchi M, Favaretto L, Bettini C, et al. (2007) Oligothiophene molecular beacons. Bioconjugate Chemistry 18: 318-322.

43. Capobianco ML, Barbarella G, Manetto A (2012) Oligothiophenes as Fluorescent Markers for Biological Applications. Molecules 17: 910-933.

44. Capobianco ML, Cazzato A, Alesi S, Barbarella G (2008) Oligothiophene-5 labeled deoxyuridines for the detection of single nucleotide polymorphisms. Bioconjugate Chemistry 19: 171-177.

45. Pieles U, Sproat BS, Neuner P, Cramer F (1989) Preparation of a Nove Psoralen Containing Deoxyadenosine Building Block for the Facile Solid-Phase Synthesis of Psoralen-Modified Oligonucleotides for a Sequence Specific Crosslink to a Given Target Sequence. Nucleic Acids Research 17: 8967-8978.

46. Pieles $U$, English U (1989) Psoralen covalently linked to oligodeoxyribonucleostides: synthesis, sequence specific recognition of DNA and photo-cross-linking to pyrimidine residues of DNA. Nucleic Acids Research 17: 285-299.

47. Lee BL, Murakami A, Blake KR, Lin SB, Miller PS (1988) Interaction of Psoralen-Derivatized Oligodeoxyribonucleoside Methylphosphonates with Single-Stranded-DNA. Biochem 27: 3197-3203.

48. Capobianco ML, Marchesi E, Perrone D, Navacchia ML (2013) Labeling Deoxyadenosine for the Preparation of Functional Conjugated Oligonucleotides. Bioconjugate Chemistry 24: 1398-1407.

49. Bosi V, Sarti E, Navacchia ML, Perrone D, Pasti L, et al (2015) Gold-nanoparticle extraction and reversed-electrode-polarity stacking mode combined to enhance capillary electrophoresis sensitivity for conjugated nucleosides and oligonucleotides containing thioether linkers. Analytic Bioanalytic Chemistry 407: 5405-5415

50. Amidites for Click Chemistry. 